\title{
EDITORIALS
}

\section{Conversations About Treatment at the End of Life}

\author{
Ben A. Rich, JD, $P h D^{7}$, and Debora A. Paterniti, $P h D^{2}$ \\ 'Bioethics Program, Department of Internal Medicine, University of California, Davis, Sacramento, CA, USA; '2Departments of Internal Medicine \\ and Sociology, University of California, Davis, Sacramento, CA, USA.
}

J Gen Intern Med 26(6):568-9

DOI: $10.1007 / \mathrm{s} 11606-011-1694-9$

(c) Society of General Internal Medicine 2011

$\mathrm{C}$ onventional wisdom in medicine maintains that most physicians, certainly those experienced in talking with patients or their proxies about code status, can profoundly influence the outcome of code status discussions. While there may be few if any studies to substantiate the conventional wisdom, there are probably more than a few grains of truth to it. The study by Downar and colleagues ${ }^{1}$ provides interesting insights into the thoughts and perspectives of patients who have elected either a "full code" (FC) or "do not resuscitate" (DNR) status, enhancing information gleaned from the dearth of earlier studies. Previous observations indicate that the likelihood of a patient opting for a "full code" status drops dramatically when the patient is fully apprised of the prognosis, especially when life expectancy is less than 6 months. ${ }^{2}$ Also influential in decreasing a patient's desire for FC status is that the patient understands that cardiopulmonary resuscitation (CPR) is unlikely to successfully restore them to an acceptable minimum level of function and quality of life. ${ }^{3}$

The findings of Downar and colleagues ${ }^{1}$ suggest that patients could benefit from discussions about end of life care during visits with their primary care physicians (PCPs) prior to having to consider code status in the hospital. Downar and colleagues $^{1}$ note that medical inpatients desiring "full code" status expressed surprise when physicians initiated the code status conversation; some even felt the conversation should not have taken place. For patients who may never have considered dying, those more hopeful about their prognosis, or those who might expect that their physician would "of course" do everything possible to keep them alive, reactions of surprise and disbelief might be anticipated. Similarly, patients who may have previously considered or discussed advance care planning and end-of-life decisions may be grateful that a physician had not simply presumed that they would wish to be "full code" and receive CPR when in fact they most certainly would not.

One particularly interesting insight of the study was the markedly different perspectives of FC and DNR patients on the nature and implications of resuscitation in the event of cardiopulmonary arrest. DNR patients viewed CPR as a kind of assault and battery in which clinicians intentionally inflict pain and suffering on patients with little or no prospect of benefit and potentially grave risk of doing further harm. These

Published online March 30, 2011 patients considered the refraining from such importunate measures as allowing for a natural and peaceful death to occur. In stark contrast, FC patients viewed withholding CPR in the event of cardiopulmonary arrest as at least medical neglect or substandard care, and at worst as euthanasia. The authors conclude that these radically different views reflect significant differences in patient perceptions rather than philosophy of life or medical care. Further informing this point, the authors note that DNR patients appeared to have a much more detailed and concrete understanding of CPR than did FC patients.

The disparity in understanding between DNR and FC patients raises yet another consideration that is often a problem in medical decision making, especially in emergency and critical care settings. A critical ethical issue is how the responsible physician should further engage with a patient in order to insure that the patient's or proxy's choice of FC or DNR status is fully informed and not based upon a serious misunderstanding or misperception of the prognosis or code status situation. ${ }^{4}$ Anticipatory discussions of advanced care decision making that occur in the more familiar context of visits with primary care physicians (PCPs), and in situations such as an annual examination or routine clinical visit for a chronic condition, which typically carry lower anxiety for patients than inpatient visits, could enhance a patient's capacity to absorb, discuss, and decide upon information related to their end-of-life care.

There are two matters of importance here in responsibly addressing patient or proxy communication-related needs regarding code status: one is the matter of the discussion content; the other, a matter of discussion timing. In the matter of discussion content, clinicians, especially hospitalists, have a propensity to focus their discussions with patients on the use of clinical procedures rather than on the goals of care. Emphasizing means over ends may undermine the fiduciary responsibility of the physician to ensure adequate and informed patient or proxy understanding of the clinical situation. Furthermore, a focus on means over ends cannot effectively assist patients with clarifying and expressing their values and priorities in the face of complex and potentially lifethreatening conditions. ${ }^{5}$ Disproportionately focusing code status discussions on procedural interventions, especially in contexts such as emergency departments and critical care units where sophisticated medical technology and an obsessive focus on procedures are ubiquitous, can erroneously convey to frightened and vulnerable patients that a DNR order will result in physician abandonment of the patient to die alone, possibly in pain or severe symptom distress.

The study authors make critically important points with regard to the timing and the novelty of code status discussions. Because it is still lamentably the case that timely and robust 
advance care planning in the primary care setting among adults without major life-threatening or limiting illness is rare, patients reasonably associate the introduction of such topics during a hospitalization as a strong indication that the situation is dire. Introduced in the primary care setting, discussions of advance directives, including code status, allow patients to absorb and process information related to code status and to consider it in the context of personal and familial belief systems, values, and preferences. Such timing provides patients greater opportunity to fully consider their decision and to think deeply and somewhat more dispassionately about their authentic wishes.

Primary care physicians (PCPs), who tend to have the closest relationships with their patients, have a unique opportunity to maximize patient expression of preferences for care. PCPs can exercise their fiduciary responsibility in exploring with a patient a patient's authentic goals for clinical care and its outcomes. Importantly, PCPs can enter into a conversation about code status with a patient before it is critical. The admonition by a group of prominent clinicians over 20 years ago remains as compelling as ever:

Physicians have a responsibility to consider timely discussions with patients about life-sustaining treatment and terminal care...as a matter of routine, physicians should become acquainted with their patients' personal values and wishes and should document them... Such discussions and documentation should be considered part of the minimal standard of acceptable care. ${ }^{6}$
Such an approach would minimize the likelihood that code status decisions are presented to patients when they may be the least capable of considering them and communicating an authentic choice.

Corresponding Author: Debora A. Paterniti, PhD; Departments of Internal Medicine and Sociology, University of California, Davis, 4150 V Street, PSSB Suite 2500, Sacramento, CA 95817, USA (e-mail: dapaterniti@ucdavis.edu).

\section{REFERENCES}

1. Downar J, Luk T, Sibbald RW, Santini T, et al. Why do patients agree to a "do not resuscitate" or "full code" order?: Perspectives of medical inpatients. JGIM. 2011; vol: pp.

2. Weeks JC, Cook EF, O'Day SJ, Peterson LM, et al. Relationship between cancer patients' predictions of prognosis and their treatment preferences. JAMA. 1998;279:1709-14.

3. Murphy DJ, Burrows D, Santilli S, Kemp AW, et al. The influence of the probability of survival on patients' preferences regarding cardiopulmonary resuscitation. N Engl J Med. 1994;330:545-9.

4. Christakis NA. Death foretold: prophecy and prognosis in medical care. University of Chicago Press. 1999.

5. Fins JJ, Miller FG, Acres CA, et al. End-of-life decision making in the hospital: current practices and future prospects. J Pain Symptom Manage. 1999;17:6-15.

6. Wanzer SH, Adelstein SJ, Federman DD, Cassel CK, et al. The physician's responsibility toward hopelessly ill patients-a second look. N Engl J Med. 1989;320:844-9. 